\title{
Determinants of Success in EGFR Mutation Status Analysis in EBUS-TBNA Specimens: The Role of PET-CT
}

\author{
Alvin Hon Man Tung*, Jenny Chun Li Ngai, Susanna So Shan Ng, Kin Wang To and Fanny Wai San Ko
}

Department of Medicine and Therapeutics, Prince of Wales Hospital, Shatin, Hong Kong

\begin{abstract}
In our Chinese cohort of 27 Non-Small Cell Lung Cancer (NSCLC) patients with mediastinal nodal involvement who underwent EBUS-TBNA, we showed no role of SUV from PET-CT scans in determining EGFR mutation status. Nonetheless, we showed that patients with high SUV $(>9.6)$ as well as number of passes could predict inadequacy of cellular material in obtaining EGFR mutation status in EBUS-TBNA.
\end{abstract}

Keywords: Endoscopic ultrasound-guided fine needle aspiration; Epidermal growth factor receptor, Positron emission tomographycomputer tomography, Bronchoscopy

\section{Case Report}

Endobronchial Ultrasound guided Transbronchial Needle Aspiration (EBUS-TBNA) samples have been shown to be useful in Epidermal Growth Factor Receptor mutation analysis in patients with Non-Small Cell Lung Cancer (NSCLC) [1,2]. There are conflicting data on links between Standard Uptake Value (SUV) recorded in Positron Emission Tomography-Computer Tomography (PET-CT) of lung tumors and EGFR mutation status [3-5]. We performed a retrospective review of all patients undergoing EBUS-TBNA in our institution to search for determinants of EGFR mutation status, and factors affecting its yield in our cohort.

Between January 2012 and March 2013, 93 patients underwent linear EBUS-TBNA in our institution. Case files of these patients were reviewed following approval from the Head of Department with demographic and clinical data retrieved. Rapid On-Site cytological Examination (ROSE) was unavailable. EGFR testing was performed using DNA PCR amplification techniques on paraffin cell-blocks made from the previously sampled specimens to determine mutations in exons $18-21$

We found 58 patients with NSCLC. 22 (46.55\%) patients underwent testing for EGFR mutation status, including 14 smokers and 15 females, with a mean age of 67 years (35-84). The average lesion sizes, number of passes, FEV1 and duration of procedure were $2.4 \mathrm{~cm}$ (1-9.0 cm), $2.9(1-4), 1.64 \mathrm{~L} / \mathrm{min}(0.72-3.20 \mathrm{~L} / \mathrm{min})$ and 41.3 minutes respectively. 12 patients were tested positive for EGFR mutation, 9 were negative whereas 6 had their tests rejected. Our EGFR mutation rate was $57 \%$, with a yield of $78 \%$. Six patients had mutations in exon 21, 5 in exon 19 and 1 in exon 20. 14 patients underwent PET-CT with SUV data of the sampled sites available. Bivariate correlation analysis using Spearman's correlation showed that females $(r=0.523, p=0.015)$, never or light smokers $(\mathrm{r}=0.523, \mathrm{p}=0.015)$ were the predictors of EGFR mutation status. Lymph node sizes $(r=0.292, p=0.2)$, number of passes $(\mathrm{r}=0.47, \mathrm{p}=840)$, FEV1 $(\mathrm{r}=-0.174, \mathrm{p}=0.610)$, duration of procedure $(\mathrm{r}=0.48, \mathrm{p}=0.837)$ and SUV on PET-CT $(\mathrm{r}=0.140, \mathrm{p}=0.700)$ were not correlated to EGFR mutation status. For the predictors of success in EGFR testing, number of passes $(\mathrm{r}=-0.463, \mathrm{p}=0.015)$ and SUV on PETCT $(r=0.635, p=0.02)$ were the only predictors in our cohort. Using the Receiver Operations Characteristics (ROC) curve. We found that with a SUV cut-off of 9.6 or more conferred a sensitivity of $66.7 \%$ with a specificity of $80 \%$, with an AUC of 0.933 (95\% CI 0.782-1.084, p=0.028) in predicting rejection in EGFR testing for mutation status.

Our results showed that the number of EBUS-TBNA passes as well as SUV scores were important determinants of success in establishing EGFR mutation status in patients with NSCLC. SUV scores were not predictive of EGFR mutation status but high SUV could potentially lead to rejected testing. The mechanism of this remains unproven, but previous data [5] suggested that a higher SUV correlates higher proliferation rates in tumor cells but not necrotic tissue. In a heterogeneous lesion, a higher SUV may also be associated with patchy areas of necrosis that were punctured by the EBUS-TBNA needle during sampling. More data are needed to confirm this phenomenon and to provide potential mechanisms for explanation.

\section{References}

1. Garcia-Olive I, Monso E, Andreo F, Sanz-Santos J, Taron M (2010) Endobronchial ultrasound-guided transbronchial needle aspiration for identifying EGFR mutations. Eur Respir J 35: 391-395.

2. Nakajima T, Yasufuku K, Nakagawara A, Kimura H, Yoshino I (2011) Multigene mutation analysis of metastatic lymph nodes in non-small cell lung cancer diagnosed by endobronchial ultrasound-guided transbronchial needle aspiration. Chest 140: 1319-1324.

3. Putora PM, Früh M, Müller J (2013) FDG-PET SUV-max values do not correlate with epidermal growth factor receptor mutation status in lung adenocarcinoma. Respirology 18: 734-735.

4. Huang CT, Yen RF, Cheng MF, Hsu YC, Wei PF, et al. (2010) Correlation of $\mathrm{F}-18$ fluorodeoxyglucose-positron emission tomography maximal standardized uptake value and EGFR mutations in advanced lung adenocarcinoma. Med Oncol 27: 9-15.

5. Kubota K, Kubota R, Yamada S (1993) FDG accumulation in tumor tissue. J Nucl Med 34: 419-421.

${ }^{*}$ Corresponding author: Alvin Hon-Man Tung, Department of Medicine and Therapeutics, Chinese University of Hong Kong, Shatin, Hong Kong, Tel: (852) 2632 3128; Fax: (852) 2649 9957; E-mail: alvin@UNSWalumni.com

Received May 23, 2014; Accepted July 23, 2014; Published July 25, 2014

Citation: Tung AHM, Ngai JCL, Ng SSS, To KW, Ko FWS (2014) Determinants of Success in EGFR Mutation Status Analysis in EBUS-TBNA Specimens: The Role of PET-CT. J Clin Case Rep 4: 395. doi:10.4172/2165-7920.1000395

Copyright: (c) 2014 Tung AHM, et al. This is an open-access article distributed under the terms of the Creative Commons Attribution License, which permits unrestricted use, distribution, and reproduction in any medium, provided the original author and source are credited. 